\title{
Technical note: Evaluation of a wireless pulse oximeter for measuring arterial oxygen saturation and pulse rate in newborn Holstein Friesian calves
}

\author{
P. Kanz, S. Krieger, M. Drillich, and M. Iwersen ${ }^{1}$ \\ Clinical Unit for Herd Health Management in Ruminants, University Clinic for Ruminants, Department for Farm Animals \\ and Veterinary Public Health, University of Veterinary Medicine Vienna, 1210 Vienna, Austria
}

\begin{abstract}
Pulse oximetry is a well-established technique in human and veterinary medicine. In farm animals, it could also be a useful tool for the detection of critical conditions relating to oxygen supply and the cardiovascular system. Among other uses, an innovative application could be the monitoring of fetuses during birth. This could help in the early identification of critical situations and support farmers and veterinarians in their decision to start obstetric or life-support interventions. Until now, however, its use in ruminant medicine was still limited to experimental applications. The objective of this study was to evaluate the accuracy of the Radius-7 Wearable Pulse CO-Oximeter (Masimo Corporation, Irvine, CA) for monitoring vital parameters in newborn calves. All measurements were conducted on animals in the lying down position. The sensor of the pulse oximeter was placed in the interdigital space of the calves' front legs and fixed with a homemade latex hoof cover. The pulsoximetric measurements of arterial oxygen saturation $\left(\mathrm{SpO}_{2}\right)$ in 40 newborn calves were compared with the corresponding results $\left(\mathrm{SaO}_{2}\right)$ from a portable blood gas analyzer (VetScan iStat1, Abaxis Inc., Union City, CA), which served as the reference. For this, an arterial blood sample was taken from the medial intermediate branch of the caudal auricular artery. In addition, the pulse rate was measured in 10 calves aged between 0 and $7 \mathrm{~d}$ with the pulse oximeter and simultaneously with a heart rate belt (Polar Equine Belt, Polar Electro Oy, Kempele, Finland) to determine their level of agreement. Spearman correlation coefficient for oxygen saturation was $93.8 \%$ for the pulse oximeter and the blood gas analyzer, and $97.7 \%$ for the pulse rate measured with the pulse oximeter and the heart rate belt. Bland-Altman plots revealed an overestimation of $\mathrm{SaO}_{2}$ by $2.95 \pm 6.39 \%$ and an underestimation of the pulse rate by $-0.41 \pm 3.18$ beats per minute compared
\end{abstract}

Received December 9, 2017.

Accepted March 11, 2018.

${ }^{1}$ Corresponding author: Michael.Iwersen@vetmeduni.ac.at with the corresponding reference methods. In summary, the pulse oximeter is suitable for continuous monitoring of arterial oxygen saturation and pulse in newborn Holstein Friesian calves. For practical use, purpose-built technical equipment is required to attach the sensor to the calves' legs.

Key words: calf, pulse oximetry, vital parameter, monitoring

\section{Technical Note}

Pulse oximetry is a noninvasive and easy-to-use technique for monitoring vital parameters such as oxygen saturation and pulse rate in human and animals. In human medicine, this technique was implemented in the 1980s and became an essential monitoring tool in emergency medicine, anesthesia, and obstetrics (Eichhorn et al., 1986; Bowes et al., 1989). Although pulse oximetry is widely used in small animal medicine, its application in farm animals is, except for some experimental uses (Uystepruyst et al., 2000; Bleul and Kähn, 2008), still not common. One issue is that no devices are available on the market that allow a practical and easy application of the technique, including attachment of the device to the animals as well as wireless transmission of the data to a display. Further aspects include the relatively high cost of the devices and uncertain economic benefits. On the other hand, commonly used techniques for detection of vital parameters, such as the auscultation of the heart or the palpation of the pulse, provide information only at the time of examination but do not allow continuous monitoring over a period of time. Thus, critical conditions that occur only for a short period of time, such as temporary decrease of oxygen saturation, can be detected with a continuous monitoring rather than by short time measurement. Among other methods of fetal and newborn monitoring (reviewed by Poulsen and McGuirk, 2009), measurement of oxygen saturation in arterial blood samples using a blood gas analyzer is suitable for gaining information about postnatal vital parameters (Bleul and Gotz, 2014). This method, however, provides information on 
the saturation only at the time of sampling and does also not allow continuous monitoring of this parameter (Chaffin et al., 1996). Furthermore, it is more invasive and difficult to perform than pulse oximetry. Therefore, pulse oximetry monitoring could be appropriate for early detection of hypoxia, especially in calves during high risk situations, such as during and after birth, and in anaesthetized animals or patients suffering from pulmonary disease. Critical conditions could be detected at an early stage so that appropriate treatment could be initiated. In case of low oxygen saturation during calving, assistance or a cesarean section may be required, whereas in newborn calves with poor vital parameters further clinical examination should be performed. All this requires a pulse oximeter validated for use in calves and a practicable method for attaching the sensor. A stable physical connection is required between the sensor and the hoof, which is necessary for reliable measurements, as well as a simple method for attaching the sensor that requires little practice. The sensor used in this study was previously tested by the manufacturer on adult humans. The objective of this study was to evaluate the accuracy of a pulse oximeter (Radius-7 Wearable Pulse CO-Oximeter, Masimo Corporation, Irvine, CA) for measuring arterial oxygen saturation $\left(\mathrm{SpO}_{2}\right)$ and pulse rate. Results were compared with the corresponding results from a portable blood gas analyzer and a heart rate belt, respectively.

The study was approved by the institutional ethics and animal welfare committee and the national authority according to $\S \S 26$ of Animal Experiments Act, Tierversuchsgesetz 2012 - TVG 2012 (GZ $68.205 / 0114-\mathrm{WF} / \mathrm{V} / 3 \mathrm{~b} / 2015$ ), as well as by the Slovakian Regional Veterinary Food Administration.

The study was conducted between May and August 2016 on a Slovakian dairy farm housing approximately 2,700 Holstein Friesian cows, and on the Teaching and Research Farm of the University of Veterinary Medicine, Vienna, Austria, housing 70 dairy cows (Simmental, Holstein Friesian, and Brown Swiss). On both farms, calving took place in straw-bedded calving pens. Calves were separated from their dam within $1 \mathrm{~h}$ of birth and housed in individual calf hutches. The wireless Radius-7 pulse oximeter was used to measure the $\mathrm{SpO}_{2}$ and the pulse rate in Holstein Friesian calves.

To evaluate the eligibility of the pulse oximeter for measuring $\mathrm{SpO}_{2}$ in calves, 40 newborn calves with no signs of life-threatening conditions were tested within 30 min of birth. A transflectance sensor (M-LNCS TF-I A, Masimo Corporation) was placed on the pars axialis of the medial hoof on one front leg of the calf. To attach the sensor to the hoof, a homemade hoof-shaped black latex cover was produced and this was slipped over the claw (Figure 1). This reusable cover was manufactured using a cast taken from the hoof of a newborn calf. The thickness of the latex was approximately 0.5 to 1 $\mathrm{mm}$. The cover contained a slit-like vertical opening at the pars axialis of the cast for insertion of the sensor and to keep it in the correct position. The sensor cable was connected to a wearable pulse oximeter for wireless transmission of data via Bluetooth to the Masimo Root device (Masimo Root, Masimo Corporation). The measured $\mathrm{SpO}_{2}$ levels, as well as the pulse rate, were visualized on the digital screen of the device and corresponding data were saved every $2 \mathrm{~s}$ on the root device. As a reference method, the arterial oxygen saturation $\left(\mathrm{SaO}_{2}\right)$ of the calf was determined using a blood gas analyzer (VetScan iStat1, Abaxis Inc., Union City, CA) that was previously evaluated in newborn calves (Bleul and Gotz, 2014). After attaching the pulse oximeter to the calf, a small area of skin $(1.5 \times 4 \mathrm{~cm})$ on the dorsal aspect of the pinna was shaved and an arterial blood sample was obtained from the medial intermediate branch of the caudal auricular artery using a heparinized syringe with a 23-gauge needle (Gaslyte arterial blood sampler, Vital Signs Inc., Englewood, CO). The blood sample was analyzed within 2 min using the portable blood gas analyzer. The analyzed oxygen saturation levels were recorded on a data-capture form. For comparison of $\mathrm{SpO}_{2}$ (pulse oximeter) and $\mathrm{SaO}_{2}$ (blood gas analyzer), mean pulse oximetry data during the last $20 \mathrm{~s}$ of blood sampling were used.

To validate the measured pulse rate of the oximeter, 10 Holstein Friesian calves aged 0 to $7 \mathrm{~d}$ and free of any signs of disease were used. For this, the sensor of the pulse oximeter was connected to each calf as described above. As the sensor was located on the hoof of the calf, measurements were performed on animals that were calm and lying down. Simultaneously, a heart rate belt (Polar Equine Belt, Polar Electro Oy, Kempele, Finland) was secured around the thorax of the calf and served as a reference method. Prior to this, the thorax was moistened with water to ensure a good contact and to obtain a valid signal from the belt. The sensor data of the belt were sent by wireless transmission to a wearable clock-like device (Polar V800, Polar Electro Oy) where data were processed, displayed, and stored. The duration of the measurement period varied between calves and was dependent on the length of time the calf was lying down. The heart-rate belt provided beat-to-beat interval data. These data were corrected for artifacts by using the correction tool of Kubios HRV software (version 2.2, Biosignal Analysis and Medical Imaging Group, Kuopio, Finland) and were then converted to heart rate values (beats per minute; bpm). To minimize the influence of potential outliers, data generated by the pulse oximeter and the heart-rate belt system were split into intervals of $20 \mathrm{~s}$ and the average 
bpm for each interval were calculated. Every interval overlapped for $10 \mathrm{~s}$ with the previous and the subsequent intervals. In this way, 1,090 measurement pairs were calculated and statistically analyzed.

Sample size calculations were performed using $\mathrm{G}^{*}$ Power (version 3.1.9.2, University of Kiel, Germany; effect size $=0.6, \alpha=0.05$, power $=0.95$ ) and further statistical evaluations were performed using SPSS Statistics for Windows (version 23.0; IBM Deutschland GmbH, Ehningen, Germany), BiAS for Windows (version 11.03, Epsilon-Verlag, Darmstadt, Germany), and Microsoft Excel 2010 (version 14.0.7180.5002, Microsoft Corporation, Redmond, WA). The level of significance was set at $P=0.05$ for all statistical tests. To compare the measurements of the pulse oximeter with the results from the blood gas analyzer $\left(\mathrm{SaO}_{2}\right)$ and the heart rate belt (for the pulse rate), Spearman's rho $(\rho)$ correlation coefficients and concordance correlation coefficients $\left(\boldsymbol{\rho}_{\mathbf{c}}\right)$ were calculated. To investigate agreement between the test results from the pulse oximeter and the blood gas analyzer, as well as the pulse oximeter and the heart rate belt, the method described by Bland and Altman (1986) was used. The cumula- tive sum test (Cusum) was performed to check data for linearity. Furthermore, potential systematic and proportional differences in the data sets were evaluated using the method recommended by Passing and Bablok (1983). Therefore the slope and the intercept of the linear regression line of the data set were calculated. No proportional differences are detected when the $95 \%$ confidence interval contains the value 1 . No systematic differences are detected if the $95 \%$ confidence interval contains the value 0 .

The arterial oxygen saturation in the 40 tested calves ranged between 15 and $98 \%$ when determined using the blood gas analyzer and between 15 and $97 \%$ when using the pulse oximeter.

The Spearman correlation coefficient $(\rho)$ for the results obtained in the reference test and the pulse oximeter was $93.8 \%(P<0.01)$. The $\rho$ values within the different classes of oxygen saturation level are shown in Table 1.

The $\rho_{\mathrm{c}}$ was $97.0 \%$ for both the saturation measurement and the pulse rate measurement.

The pulse rate in 10 calves was observed for an average period of time of approximately 18 min (minimum 14

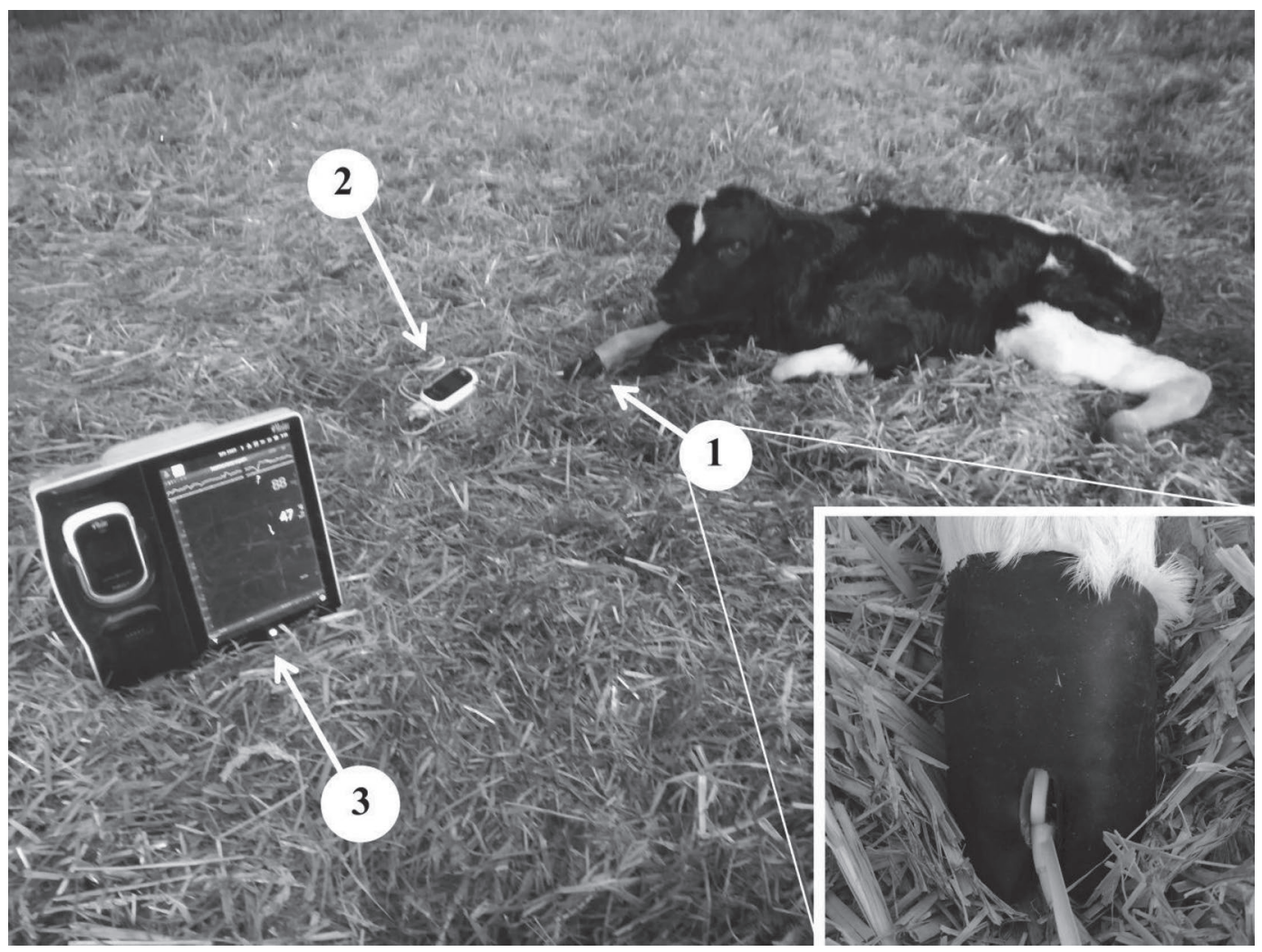

Figure 1. Technical equipment of the pulse oximeter and attachment of the sensor to the calf. (1) Latex hoof cover with transflectance sensor (M-LNCS TF-I A, Masimo Corporation, Irvine, CA) located in the interdigital space of the calf. (2) Cable link to the wearable pulse oximeter (Radius-7 Wearable Pulse CO-Oximeter; Masimo Corporation). (3) Masimo Root (Masimo Corporation) with digital display for visualizing the measured parameters. 
Table 1. Differences between oxygen saturation measured with the Masimo pulse oximeter and the reference method (blood gas analyzer) and pulse rates measured with the pulse oximeter and the heart rate belt using the Passing-Bablok and Bland-Altman analyses

\begin{tabular}{|c|c|c|}
\hline \multirow[b]{2}{*}{ Item } & \multirow{2}{*}{$\begin{array}{c}\text { Arterial oxygen saturation } \\
\text { Pulse oximeter vs. } \\
\text { blood gas analyzer }\end{array}$} & \multirow{2}{*}{$\begin{array}{l}\text { Pulse rate } \\
\text { ulse oximeter vs. } \\
\text { heart rate belt }\end{array}$} \\
\hline & & \\
\hline $\begin{array}{l}\text { Overall Spearman correlation } \\
\text { coefficient } \rho(\%)\end{array}$ & 93.8 & 97.7 \\
\hline$<31 \%^{1,2}$ & 65.4 & \\
\hline $31-70 \%{ }^{1}$ & 96.2 & \\
\hline$>70 \%$ & 72.8 & \\
\hline \multicolumn{3}{|l|}{ Passing-Bablok } \\
\hline Slope $(b)$ & 1.00 & 1.00 \\
\hline $\mathrm{CI}_{95}^{4}$ for $b$ & 0.95 to 1.06 & 0.99 to 1.00 \\
\hline Intercept $(a)$ & 2.00 & 0.60 \\
\hline $\mathrm{CI}_{95}$ for $a$ & -1.44 to 3.52 & -1.71 to 1.73 \\
\hline \multicolumn{3}{|l|}{ Bland-Atman $( \pm \mathrm{SD})$} \\
\hline Overall bias & $2.95 \pm 6.39$ & $-0.41 \pm 3.18$ \\
\hline$<31 \%$ & $3.75 \pm 7.51$ & \\
\hline $31-70 \%$ & $2.56 \pm 5.41$ & \\
\hline$>70 \%$ & $2.32 \pm 5.95$ & \\
\hline \multicolumn{3}{|c|}{${ }^{1}$ Classification refers to the results of the blood gas analyzer. } \\
\hline \multicolumn{3}{|c|}{$\begin{array}{l}{ }^{2} \text { Referring to human medicine, where oxygen saturation levels of }>30 \% \text { are seen as an adequate supply for the } \\
\text { fetus (Dildy, 2001). } \\
{ }^{3} \text { Cut-off value referring to a range of } 70 \text { to } 100 \% \text {, reported in adult human patients by the manufacturer using } \\
\text { this sensor type. } \\
{ }^{4} \mathrm{CI}_{95}=95 \% \text { confidence interval. }\end{array}$} \\
\hline
\end{tabular}

min, maximum $29 \mathrm{~min})$. The pulse rate ranged between 94 and $185 \mathrm{bpm}$ measured with the belt and between 93 and $180 \mathrm{bpm}$ measured with the pulse oximeter. The corresponding Spearman correlation coefficient for all calves was $97.7 \%(P<0.01)$. The Spearman correlation coefficient for each calf varied between 71.2 and $98.3 \%$.

The Bland-Altman plot showed that the pulse oximeter overestimated the mean $\mathrm{SaO}_{2}$ levels measured using the blood gas analyzer by $2.95 \pm 6.39 \%$, whereas the mean pulse rate was underestimated (negative bias) by the pulse oximeter by $-0.41 \pm 3.18 \mathrm{bpm}$ compared with the heart rate belt. The biases \pm standard deviations stratified for the different saturation classes are presented in Table 1.

The cumulative sum test detected no significant deviation from linearity for both the oxygen saturation $(P$ $=0.85)$ and the pulse rate $(P=0.09)$ testing methods, showing the Passing-Bablok method to be reliable for statistical analyses.

According to the Passing-Bablok analyses (Table 1), neither proportional nor systematic differences were detected for either the oxygen saturation or the pulse measurements, as the confidence intervals for the slopes contain the value 1 and the confidence intervals for the intercepts contain the value 0 .

Additional descriptive parameters for oxygen saturation measurements as well as for pulse rate measurements are presented in Tables 2 and 3, respectively.
To our knowledge, the interdigital space has not been used previously for obtaining vital parameters in calves. We have chosen this site for future use of the device for monitoring vital parameters of calves during stage II of labor under field conditions. The interdigital space can be reached during the calving process earlier than other possible sites, such as the nose or ear.

Strong movements of the extremity that is used for attachment of the sensor present the risk of displacement of the sensor, leading to artifacts and inaccurate data (Jubran, 2015). Hence, the duration of a valid monitoring period depended on the length of time the calf remained calm and in a prone position. As soon as the calf attempted to stand, the measurements were stopped. Thus, strictly speaking, the results of this study can be considered as valid only for calm calves that are lying down. Additionally, the degree of pigmentation of the tissue on which the sensor is placed and the influence of ambient light may also have a negative effect on the accuracy of the measurements (Fluck et al., 2003; Bickler et al., 2005). Therefore, all calves that were used in this study had comparable, faintly pigmented hoofs. To prevent interference by ambient light, the hoof cover was made from opaque latex material. Further studies should evaluate the influence of parameters such as movement of the patient during measurement, hoof pigmentation, as well as breed and age of the patient. 
Table 2. Descriptive statistics of the arterial oxygen saturation tested in 40 calves performed immediately after birth using the Masimo pulse oximeter and using the VetScan iStat1 blood gas analyzer ${ }^{1}$

\begin{tabular}{|c|c|c|}
\hline \multirow[b]{2}{*}{ Parameter } & \multicolumn{2}{|c|}{ Arterial oxygen saturation } \\
\hline & Pulse oximeter & Blood gas analyzer \\
\hline \multicolumn{3}{|c|}{ No. of samples } \\
\hline Total & 40 & 40 \\
\hline$<31 \%^{2}$ & 12 & 16 \\
\hline $31-70 \%$ & 13 & 9 \\
\hline$>70 \%^{3}$ & 15 & 15 \\
\hline \multicolumn{3}{|c|}{ Mean \pm SE $(\%)$} \\
\hline Total & $54.85 \pm 4.92$ & $51.90 \pm 4.92$ \\
\hline$<31 \%$ & $21.58 \pm 1.26$ & $22.50 \pm 1.37$ \\
\hline $31-70 \%$ & $43.15 \pm 3.27$ & $41.89 \pm 3.77$ \\
\hline \multirow{2}{*}{\multicolumn{3}{|c|}{ Median \pm interquartile range $(\%)$}} \\
\hline & & \\
\hline Total & $42.00 \pm 64.50$ & $37.50 \pm 62.50$ \\
\hline$<31 \%$ & $20.50 \pm 7.5$ & $23.50 \pm 10.75$ \\
\hline $31-70 \%$ & $40.00 \pm 13.00$ & $38.00 \pm 16.50$ \\
\hline$>70 \%$ & $92.00 \pm 6.00$ & $91.00 \pm 12.00$ \\
\hline
\end{tabular}

In this study, a stable signal was provided by the pulse oximeter and by the heart rate belt for all calves. This means that no disconnection of the sensor or signal loss occurred during the measurement period, which is an important requirement for the use under field conditions. Future studies should evaluate the fixation of the pulse oximeter under different field conditions.

The detected $\rho_{\mathrm{c}}$ of $97.0 \%$ for both parameters measured using the pulse oximeter, and using the corresponding reference method, were classified as substantial according to McBride (2005). The overall $\rho$ was very strong $(93.8 \%)$ between both methods for measuring the oxygen saturation, but the pulse oximeter tended to produce overestimated results compared with the blood gas analyzer. A different model of pulse oximeter was evaluated in calves by Bleul and Kähn (2008), resulting in a bias of $-3.9 \pm 7.4 \%$. Koenig et al. (2003) reported that the pulsoximetric measurements underestimates $\mathrm{SaO}_{2}$ by $3.6 \pm 3.2 \%$ and $3.1 \pm 5.2 \%$ when tested on horses with values ranging from 95 to $100 \%$ and 91 to $100 \% \mathrm{SaO}_{2}$, respectively. Reported biases in dogs ranged from $-0.83 \pm 2.60 \%$ to $1.52 \pm 1.62 \%$, and for cats from $-4.26 \pm 9.81 \%$ to $2.32 \pm 5.07 \%$, depending on the type of the pulse oximeter used (Matthews et al., 2003). For pulse oximeters in human medicine, biases of $<2 \%$ and standard deviations of $<3 \%$ were reported when tested in healthy adults with $\mathrm{SaO}_{2}>90 \%$ (Nickerson et al., 1988). However, accuracy decreased in patients with lower $\mathrm{SaO}_{2}$ levels (Severinghaus et al., 1989; Hannhart et al., 1991). A slight tendency for decreasing accuracy at lower $\mathrm{SaO}_{2}$ levels was also detected in our study. Compared with the accuracy of pulse oximetry evaluated in human medicine, the bias we detected in calves was only marginally higher, although $60 \%$ of the animals had a $\mathrm{SaO}_{2}$ of $\leq 70 \%$. Therefore, the accuracy of this method is considered as acceptable for continuous monitoring of farm animals under field conditions. The utility, however, might be affected in terms of potential threshold levels for critical conditions as suggested by others (Dildy, 2001; Bleul and Kähn, 2008).

Table 3. Descriptive statistics of the pulse rate in 10 calves aged between 0 and $7 \mathrm{~d}$ measured with the Masimo pulse oximeter and the Polar Equine Belt ${ }^{1}$

\begin{tabular}{lcc}
\hline & \multicolumn{2}{c}{ Pulse rate } \\
\cline { 2 - 3 } Parameter & Pulse oximeter & Heart rate belt \\
\hline No. of averaged 20-s intervals & 1,090 & 1,090 \\
Mean \pm SE $\left(\mathrm{bpm}^{2}\right.$ ) & $123.5 \pm 0.4$ & $123.9 \pm 0.4$ \\
Median \pm interquartile range $(\mathrm{bpm})$ & $122.4 \pm 16.2$ & $122.6 \pm 16.6$ \\
\hline${ }^{1}$ Masimo Corporation (Irvine, CA), Polar Equine Belt (Polar Electro Oy, Kempele, Finland). \\
${ }^{2}$ bpm = beats per minute.
\end{tabular}


Further research should evaluate if the threshold value for bovine fetuses of, for example, $30 \%$ saturation can be considered as valid for this type of pulse oximeter and sample site as well or if it is necessary to increase it according to the bias we detected.

The overall $\rho$ for the pulse rate measurement was very strong (97.7\%), although it varied in each calf between 71.2 and $98.3 \%$. It has to be noted that the heart rate belt used in this study has not yet been evaluated in calves, but Hopster and Blokhuis (1994) detected a correlation coefficient of 0.88 for motionless cows by using a Polar heart rate monitor and an electrocardiograph. Hence, the accuracy of the pulse measurement results in our study should be considered critically.

Using the interdigital space as the site for the sensor, in combination with the latex hoof cover, resulted in good attachment of the sensor and no signal loss. Nevertheless, it has to be considered that all calves in our study were in a sternal recumbent position and their limbs moved little during measurement. Further studies are required to evaluate the stability of sensor attachment and the signal quality in animals under more challenging conditions, such as standing and walking, as well as in bovine fetuses during parturition. The performance of the pulse oximeter is considered to be suitable for measuring the pulse rate and $\mathrm{SpO}_{2}$ levels in motionless calves when the sensor is attached to the slightly pigmented front hoof of calves.

\section{ACKNOWLEDGMENTS}

The authors thank the Jutta and Georg Bruns-Stiftung (Steinfeld, Germany) for financial support for this study and the staff of FirstFarms, Slovakia, for their assistance with logistics of the project.

\section{REFERENCES}

Bickler, P. E., J. R. Feiner, and J. W. Severinghaus. 2005. Effects of skin pigmentation on pulse oximeter accuracy at low saturation. Anesthesiology 102:715-719.

Bland, J. M., and D. G. Altman. 1986. Statistical methods for assessing agreement between two methods of clinical measurement. Lancet 1:307-310.
Bleul, U., and E. Gotz. 2014. Evaluation of the i-STAT portable pointof-care analyzer for determination of blood gases and acid-base status in newborn calves. J. Vet. Emerg. Crit. Care (San Antonio) 24:519-528.

Bleul, U., and W. Kähn. 2008. Monitoring the bovine fetus during stage II of parturition using pulse oximetry. Theriogenology 69:302-311.

Bowes, W. A. J. I., B. C. Corke, and J. Hulka. 1989. Pulse oximetry: A review of the theory, accuracy and clinical applications. Obstet. Gynecol. 74:541-546.

Chaffin, M. K., N. S. Matthews, N. D. Cohen, and G. K. Carter. 1996 Evaluation of pulse oximetry in anaesthetised foals using multiple combinations of transducer type and transducer attachment site. Equine Vet. J. 28:437-445.

Dildy, G. A. 2001. The future of intrapartum fetal pulse oximetry. Curr. Opin. Obstet. Gynecol. 13:133-136.

Eichhorn, J. H., J. B. Cooper, D. J. Cullen, W. R. Maier, J. H. Philip, and R. G. Seeman. 1986. Standards for patient monitoring during anesthesia at Harvard medical school. JAMA 256:1017-1020.

Fluck, R. R. Jr., C. Schroeder, G. Frani, B. Kropf, and B. Engbretson. 2003. Does ambient light affect the accuracy of pulse oximetry? Respir. Care 48:677-680.

Hannhart, B., J. P. Haberer, C. Saunier, and M. C. Laxenaire. 1991. Accuracy and precision of fourteen pulse oximeters. Eur. Respir. J. 4:115-119.

Hopster, H., and H. J. Blokhuis. 1994. Validation of a heart-rate monitor for measuring a stress response in dairy cows. Can. J. Anim. Sci. 74:465-474

Jubran, A. 2015. Pulse oximetry. Crit. Care 19:272.

Koenig, J., W. McDonell, and A. Valverde. 2003. Accuracy of pulse oximetry and capnography in healthy and compromised horses during spontaneous and controlled ventilation. Can. J. Vet. Res. $67: 169-174$.

Matthews, N. S., S. Hartke, and J. C. Allen Jr.. 2003. An evaluation of pulse oximeters in dogs, cats and horses. Vet. Anaesth. Analg. 30:3-14.

McBride, G. B. 2005. A proposal for strength-of-agreement criteria for Lin's Concordance Correlation Coefficient. NIWA Client Report: HAM 2005-062.

Nickerson, B. G., C. Sarkisian, and K. Tremper. 1988. Bias and precision of pulse oximeters and arterial oximeters. Chest 93:515-517.

Passing, H., and Bablok.. 1983. A new biometrical procedure for testing the equality of measurements from two different analytical methods. Application of linear regression procedures for method comparison studies in clinical chemistry, Part I. J. Clin. Chem. Clin. Biochem. 21:709-720.

Poulsen, K. P., and S. M. McGuirk. 2009. Respiratory disease of the bovine neonate. Vet. Clin. North Am. Food Anim. Pract. 25:121137.

Severinghaus, J. W., K. H. Naifeh, and S. O. Koh. 1989. Errors in 14 pulse oximeters during profound hypoxia. J. Clin. Monit. 5:72-81.

Uystepruyst, C. H., J. Coghe, F. Bureau, and P. Lekeux. 2000. Evaluation of accuracy of pulse oximetry in newborn calves. Vet. J. 159:71-76 